\title{
Evaluation of intestinal damage biomarkers in calves with atresia coli
}

\author{
Ramazan Yildiz', Mahmut $\mathrm{Ok}^{2}$, Merve Ider ${ }^{2}, \mathrm{Ugur}^{1}$ Aydogdu ${ }^{3}$, \\ Amir Naseri ${ }^{2}$, Kurtulus Parlak ${ }^{4}$, Erdem Gulersoy ${ }^{2}$ \\ ${ }^{1}$ Department of Internal Medicine, Faculty of Veterinary Medicine, \\ Mehmet Akif Ersoy University, 15030, Burdur, Turkey \\ ${ }^{2}$ Department of Internal Medicine, ${ }^{4}$ Department of Surgery, Faculty of Veterinary Medicine, \\ Selcuk University, 42003, Konya, Turkey \\ ${ }^{3}$ Department of Internal Medicine, Faculty of Veterinary Medicine, \\ Balikesir University, 10145, Balikesir, Turkey \\ ramazanyildiz@mehmetakif.edu.tr
}

Received: February 21, 2018 Accepted: August 23, 2018

\begin{abstract}
Introduction: Intestinal obstruction such as atresia coli causes pathophysiological changes in gastrointestinal tissue due to the rise of intra-abdominal pressure. The aim of this study is to determine the intestinal damage with intestinal biomarkers in calves with atresia coli. Material and Methods: The study was conducted on 40 Holstein calves diagnosed with atresia coli with mild to moderate abdominal distention and 10 healthy Holstein calves which served as the control. Blood samples were collected from all calves, and then serum concentrations of intestinal biomarkers were estimated, namely intestinal fatty acid binding protein (IFABP), liver fatty acid binding protein (LFABP), trefoil factor 3 (TFF3), and intestinal alkaline phosphatase (IAP), using commercially available specific bovine ELISA kits. An automatic blood gas analyser was employed for determining the lactate concentration. Results: The concentrations of serum LFABP $(\mathrm{P}<0.01)$, IFABP, TFF3, IAP, and blood lactate $(\mathrm{P}<0.001)$ were significantly higher in calves with atresia coli than in healthy calves. Conclusion: The calves affected with atresia coli exhibited severe intestinal damage, and IFABP, LFABP, and TFF3 have significant diagnostic importance and play a useful role in determining the intestinal damage due to intestinal obstruction. High levels of IAP and lactate may serve as a signal for the development of intestinal injury.
\end{abstract}

Keywords: calves, atresia coli, IFABP, LFABP, TFF3.

\section{Introduction}

Congenital intestinal atresia is a defect characterised by the complete closure of a segment of the intestinal tract. Colonic atresia has been reported in humans and domestic animals. In calves, intestinal atresia is identified in different segments of the intestine, including the rectum, anus, colon, and ileum $(1,5,18)$. The disease is prevalent among Holstein, Ayrshire, Shorthorn, Simmental, Hereford, Angus, and Maine Anjou breeds and in crossbred cattle. Genetic studies have suggested atresia coli in calves to have an inherited basis, and the cause of the abnormality is found to be the presence of homozygous recessive alleles of the defective gene for atresia coli $(5,28)$. Vascular insufficiency of the developing colon in calves and diagnosis during early pregnancy in cattle by amniotic palpation may damage the foetal intestinal blood supply and disrupt organogenesis, thereby increasing the risk of atresia coli (5).

Mechanical obstructions are a result of abnormalities in the bowel lumen, intestinal wall, or outside the intestinal tract. Congenital intestinal obstructions in calves, such as atresia jejuni, coli, recti, or ani, result in the inability of the calves to defecate after birth (4) and require surgical correction. Intestinal obstruction, which causes pathophysiological changes due to the rise of intra-abdominal pressure, is called abdominal compartment syndrome (ACS). Increasing intra-abdominal pressure causes progressive hypoperfusion and ischaemia of the intestines and other peritoneal and retroperitoneal structures (6). In 
newborn infants, ACS usually develops due to congenital gastrointestinal atresia, abdominal wall defects, and ileus. It is a clinical syndrome characterised by increased intra-abdominal pressure and progressive intra-abdominal organ dysfunction. An accumulation of intestinal contents in intestinal atresia or stenosis is considered to be the main cause. The impaired vascularisation usually observed in ACS, has also been reported to result in ischaemic necrosis of the intestine (26) and increased intra-abdominal pressure, causing intestinal ischaemia by reducing blood flow in the mesenteric arteries (31). Other clinical outcomes include severe gut oedema and ascites (9).

An increase in intestinal biomarkers such as intestinal fatty acid binding proteins (IFABPs) has been reported to occur in acute intestinal ischaemia and inflammatory bowel damage (13). Intestinal IFABP is only expressed by enterocytes of the small intestine (22). Another biomarker, liver-type fatty acid binding protein (LFABP) has been isolated from the intestine, liver, kidneys, and pancreas (10).

Trefoil factor 3 (TFF3) plays a key roles in mucosal protection through mucous barrier formation (16). For instance, an injury to the gastrointestinal tract like in inflammatory bowel syndrome is associated with elevated levels of TFF3 (27). Intestinal alkaline phosphatase (IAP) is an enzyme produced by apical enterocytes and released into the intestinal luminal brush border in response to enteral feeding. It is believed to play an important role in the mucosal defence of the intestines, mainly through the detoxification of lipopolysaccharides (19). The changes in the levels of these biomarkers have been reported to be indicative of gastrointestinal mucosal damage (12, 19, 21).

We hypothesised that in intestinal atresia of calves, abdominal distention could cause breakdown of the gastrointestinal mucosal layer because of derangements of intestinal microcirculation. To test this hypothesis, we decided to evaluate the role of various intestinal biomarkers to determine the occurrence of intestinal damage in the atresia coli of calves.

\section{Material and Methods}

Animals. The material for the present study derived from 40 Holstein calves diagnosed with atresia coli and mild to moderate abdominal distention (experimental group) and 10 healthy Holstein calves that served as the control group. All calves were from 2 to 5 days of age and from farms in Konya region. All of them had normal gestation and parturition processes.

Clinical examination. A routine clinical examination, consisting of evaluation of body temperature, heart rate, and respiratory rate was performed. Finger exploration through the rectum did not reveal the presence of meconium. The rectum and colon were examined with warm water using a tube, the water first being allowed to gravitate into the colon and then being forced out, showing a white mucous plug but no faecal material. All calves were examined ultrasonographically. Exploratory surgery was performed to confirm the correct diagnosis of atresia coli.

Blood sample collection. The blood samples obtained by venipuncture of the jugular vein were placed into serum tubes. The tubes were centrifuged at $5,000 \mathrm{rpm}$ for $10 \mathrm{~min}$ after clotting, and the serum was carefully harvested and stored at $-80^{\circ} \mathrm{C}$ until analysis.

Immunoassays for intestinal biomarkers. Serum concentrations of intestinal biomarkers, namely IFABP, LFABP, TFF3, and IAP, were determined using commercially available specific bovine enzyme-linked immunosorbent assay (ELISA) kits (MyBiosource, USA). The biomarkers were determined according to the manufacturer's recommendations and the results were calculated with the optimal standard curve. Absorption was recorded on an ELX800 universal microplate reader (BIOTEK, USA), and lactate concentration and oxygen saturation $\left(\mathrm{SatO}_{2}\right)$ percentage were analysed using a GEM Premier 3000 automatic blood gas analyser (Instrumentation Laboratory, USA) within $15 \mathrm{~min}$ after sampling. White blood cells were analysed with an MS4e-Vet haematology analyser (Melet Schlosing Laboratories, France) within 15 min after sampling.

Statistical analysis. The distribution of the data was determined using the Kolmogorov-Smirnov test. Only the values of IFABP and TFF3 had non-normally distributed data and the Mann-Whitney U test was used for testing the significance of the difference between the experimental and control groups. Student's $t$-test was used for statistical analyses of other parameters. Data were presented as mean values and standard errors of means (SEMs) (mean \pm SEM) in the case of Student's $t$-test and median (minimum/maximum) values in the case of the Mann-Whitney U test.

\section{Results}

The principal clinical findings were depression, weakness, poor or absence of suction reflex, dehydration, increased capillary refill time, paleness of mucous membranes, abdominal respiration, abdominal distention, palpable distended intestinal loops at the abdomen, and leg movement through to the abdomen. Rectal examination with digital palpation revealed empty rectum and the presence of mucus in the intestine. Systemic clinical changes such as elevated heart rates $(132 \pm 4$ beats $/ \mathrm{min})$ and respiratory rates $(58.1 \pm 5 / \mathrm{min})$, were observed. There was no statistical difference in body temperature between the two groups. The venous blood lactate level was significantly higher $(\mathrm{P} \leq 0.001)$ and $\mathrm{SatO}_{2}(\mathrm{P} \leq 0.01)$ level significantly lower in calves with atresia coli compared to healthy calves. When compared to the control group, the white 
Table 1. IFABP, LFABP, IAP, TFF3, lactate, $\mathrm{SatO}_{2}$, and $\mathrm{WBC}$ levels in healthy calves and calves with atresia coli

\begin{tabular}{lcc}
\hline Parameters & $\begin{array}{c}\text { Healthy calves } \\
(\mathrm{n}: 10)\end{array}$ & $\begin{array}{c}\text { Calves with atresia coli } \\
(\mathrm{n}: 40)\end{array}$ \\
\hline $\begin{array}{l}\text { IFABP }(\mathrm{ng} / \mathrm{mL})^{* * *} \\
\text { Median }(\mathrm{min} / \mathrm{max})\end{array}$ & $14.5(12.4 / 16.2)$ & $17.1(12.4 / 36.2)$ \\
\hline $\begin{array}{l}\text { LFABP }(\mathrm{ng} / \mathrm{mL})^{* *} \\
\text { Mean } \pm \mathrm{SEM}\end{array}$ & $3.18 \pm 0.3$ & $4.48 \pm 0.24$ \\
\hline $\begin{array}{l}\mathrm{IAP}(\mathrm{ng} / \mathrm{mL})^{* * *} \\
\text { Mean } \pm \mathrm{SEM}\end{array}$ & $30.0 \pm 1.33$ & $40.7 \pm 2.43$ \\
\hline $\begin{array}{l}\text { TFF3 }(\mathrm{ng} / \mathrm{mL})^{* * *} \\
\text { Median }(\mathrm{min} / \mathrm{max})\end{array}$ & $1.6(0.9 / 1.9)$ & $3.8(1.5 / 68)$ \\
\hline $\begin{array}{l}\text { Lactate }(\mathrm{mmol} / \mathrm{L})^{* * *} \\
\text { Mean } \pm \mathrm{SEM}\end{array}$ & $1.65 \pm 0.09$ & $3.88 \pm 0.39$ \\
\hline $\begin{array}{l}\text { SatO }(\%) * * \\
\text { Mean } \pm \mathrm{SEM}\end{array}$ & $55.5 \pm 6.39$ & $31.9 \pm 2.15$ \\
\hline $\begin{array}{l}\mathrm{WBC}\left(10^{3} / \mu \mathrm{L}\right)^{* *} \\
\text { Mean } \pm \mathrm{SEM}\end{array}$ & $10.4 \pm 0.97$ & $15.2 \pm 1.46$ \\
\hline
\end{tabular}

IFABP - intestinal fatty acid binding protein, LFABP - liver fatty acid binding protein, TFF3 - trefoil factor 3, IAP - intestinal alkaline phosphatase, $\mathrm{WBC}$ - white blood cells, $\mathrm{SatO}_{2}-$ oxygen saturation, $\mathrm{SEM}-$ standard error of mean, $\min / \max -\operatorname{minimum} /$ maximum

$* * \mathrm{P} \leq 0.01 * * * \mathrm{P} \leq 0.001$
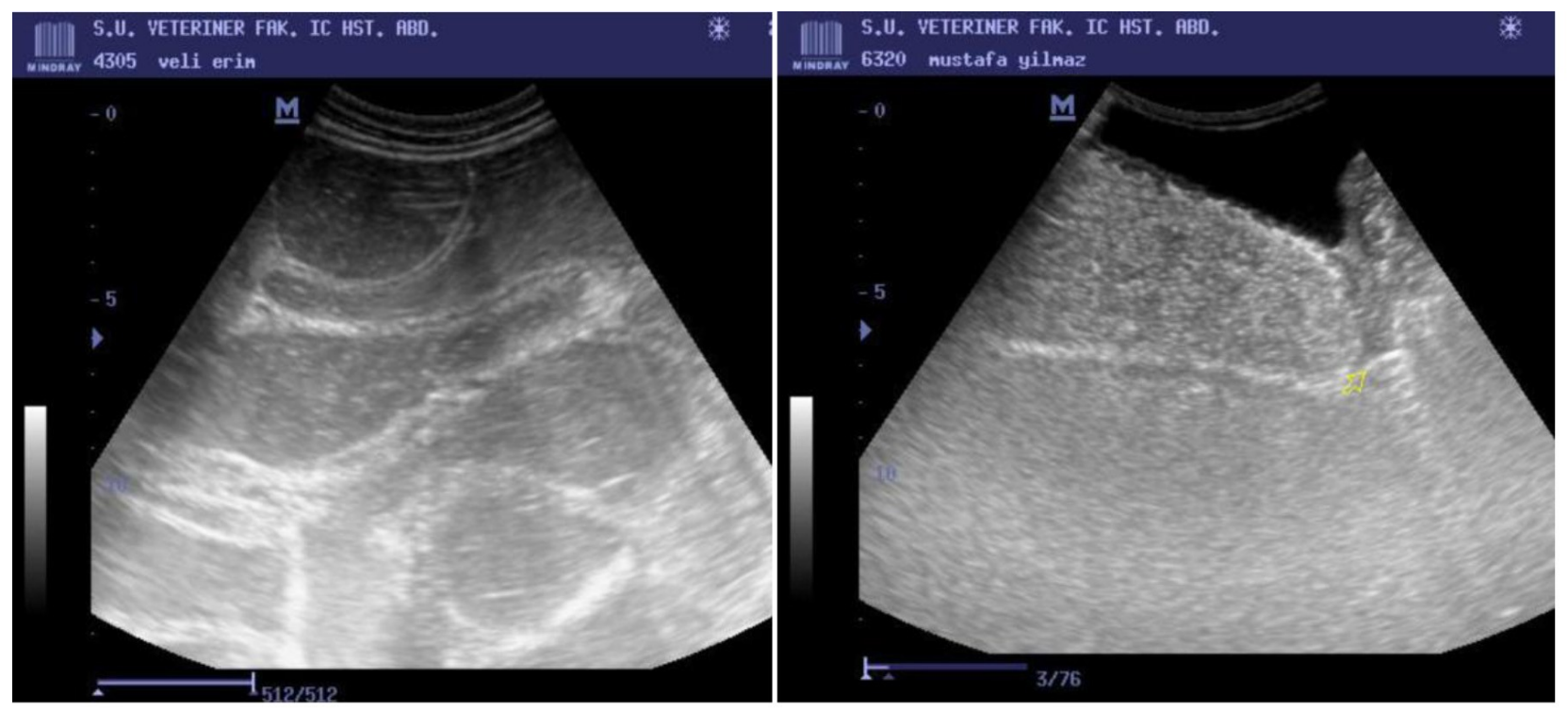

Fig. 1. Enlargement of intestinal loops in calves with atresia coli (the yellow arrow defines the suspected area of atresia in the colon)

blood cell (WBC) count was significantly higher $(\mathrm{P} \leq 0.01)$ in calves with atresia coli (Table 1). Ultrasonographic examination demonstrated that in most cases the intestines were enlarged and filled with contents (Fig. 1), and sometimes contained fibrin mesh which was floating in the abdomen.

The levels of serum IFABP, LFABP, TFF3, and IAP and blood lactate, $\mathrm{SatO}_{2}$, and WBC parameters in the calves with atresia coli and healthy calves are presented in Table 1. The serum IFABP $(\mathrm{P}<0.001)$, LFABP $(\mathrm{P}<0.01)$, IAP $(\mathrm{P}<0.001)$, and TFF3 $(\mathrm{P}<0.001)$ levels were higher in the calves with atresia coli compared to the healthy calves (Table 1).

\section{Discussion}

Numerous studies have been conducted on the role of intestinal biomarkers in human patients; however, we report their use in the diagnosis of intestinal obstruction in farm animals for the first time. We believe that the present study paves the way for further studies in this regard in farm animals.

Mucosal organs such as the intestine are supported by a rich and extensive underlying vasculature. For this reason, the intestine and particularly the barrierprotective epithelial cells are susceptible to damage related to diminished blood flow and concomitant 
tissue hypoxia (11). Obstructions to any segment in the intestine prevent the normal movement of gut content and meconium through it in the foetus and neonate, consequently leading to the dilation of the proximal part, accompanied by progressive abdominal distention. The intestinal diameter beyond the discontinuity is small and filled with mucus and desquamated cells, preventing the movement of faecal contents to the anus (2). Intestinal obstruction may in turn lead to ACS and an accumulation of fluid and gas in the intestine, culminating in mucosal and serosal oedema and capillary leaks. Damage to the mucosal barrier allows bacteria, toxins, pro-inflammatory factors, and free oxygen radicals to switch from the intra-abdominal system to the circulatory system. This may cause complications or lesions, including vascular obstruction, congestion, haemorrhage, ischaemic injury, and infarction $(6,12,15)$.

In the current study, we believe that in calves with atresia coli, intestinal obstruction leads to ACS, and complications which are related to ACS may cause intestinal damage. Biomarkers are used to detect different intestinal damage-related conditions and complications.

Elevated levels of intestinal biomarkers have been reported to be present in acute intestinal ischaemia and inflammatory bowel damage (13), and intestinal mucosal injury has been observed to be attended by an increase in IFABP, LFABP, IAP, and TFF3 levels (13, 21). Patients with gastrointestinal complications are known to develop high IFABP levels, and patients with multiple traumas show high IFABP and LFABP levels correlating with the severity of abdominal tissue damage. Both IFABP and LFABP could serve as useful markers for the early detection of significant abdominal injury (23). Uzun et al. (29) found that patients with mesenteric ischaemia and intra-abdominal mass had significantly higher IFABP levels than those in the healthy group. The mean levels of IFABP for the acute mesenteric ischaemia group, intra-abdominal mass group, and control group were 708.6, 387.4, and $61.0 \mathrm{pg} / \mathrm{mL}$, respectively. LFABP and IFABP levels significantly increased in damaged abdominal tissue in humans, signifying IFABP and LFABP as useful intestinal biomarkers for the early identification of abdominal injury. It is reported that serum IFABP sensitively reflects bowel damage in small bowel obstruction, and is considered to be a potential biomarker for detecting this condition (25). Similarly, Kittaka et al. (17) stated that it to be useful for discriminating between strangulated small bowel obstruction and simple small bowel obstruction. $\mathrm{Ng}$ et al. (21) reported that gut barrier biomarkers such as IFABP, LFABP, and TFF3 were more specific than common inflammatory mediators such as C-reactive protein (CRP) for diagnosis of necrotising enterocolitis in preterm infants. In the present study, the serum IFABP $(\mathrm{P}<0.001)$, LFABP $(\mathrm{P}<0.01)$, and lactate $(\mathrm{P}<0.001)$ levels were higher in the calves with atresia coli compared with the healthy calves. The higher values of IFABP and LFABP levels may be related to intestinal epithelium damage manifested by a reduction in tissue perfusion of the intestine because of the pressure on the intestinal wall of luminal contents.

Recently, another study proposed IFABP and LFABP serum levels to be efficient screening tools for the detection of tissue-specific injury after hypoxia and intestinal damage (15). In this study, statistically higher lactate and lower $\mathrm{SatO}_{2}$ levels were detected, implying the presence of hypoxia in the calves with atresia coli. Additionally, the statistically higher WBC counts in calves with atresia coli may be associated with peritonitis or the onset of sepsis related to bacterial overgrowth in the intestines (7). These findings are likely indicators of impaired intestinal perfusion in calves with atresia coli. Several recent studies support this hypothesis and suggest FABPs as useful indicators of tissue damage after hypoxia and intestinal injury due to their organ-specific distribution $(7,8,14)$. An elevated IFABP concentration also correlates with splanchnic hypoperfusion and mucosal damage in critically ill patients with abdominal sepsis (10).

It has been documented that hypoxia increases expression of TFF3 in intestinal epithelial cells and is interpreted as a mechanism for the protection of the barrier function when oxygen levels are low (11). Srivastava et al. (27) showed that serum TFF3 was significantly higher in patients with ulcerative colitis than in healthy volunteers, and the TFF3 parameter could be used as a potential biomarker to assess mucosal healing in such patients. Zurek et al. (32) demonstrated a strong relationship between gut mucosal damage, measured by TFF3 level, and severity of disease. The expression of TFF3 increased for $6 \mathrm{~h}$ after cecal ligation and puncture and decreased at 24 and $48 \mathrm{~h}$ later (3). Another biomarker, IAP is thought to be involved in gut mucosal defence through the detoxification of lipopolysaccaride (12). However, Lapsekili et al. (20) reported an insignificant contribution of IAP in the diagnosis of acute mesenteric ischaemia. Using systemic IAP appears to be effective in alleviating the systemic inflammatory response associated with enterocolitis and may be accepted as a useful adjunctive treatment for necrotising enterocolitis (24). In the current study, the serum IAP $(\mathrm{P}<0.001)$ and TFF3 $(\mathrm{P}<0.001)$ levels were higher in the calves with atresia coli compared with the healthy calves. The reason for the increase in IAP and TFF concentrations may be related to the repair of intestinal ischaemic injury or prevention of mucosal damage, as pointed out by a study on the essential role TFF3 and IAP in mucosal protection and repair $(24,30)$.

In conclusion, the results of the present study indicate that calves with atresia coli suffer from intestinal damage related to abdominal distention. This observation should be considered while designing the treatment modality or taking the surgical decision. Intestinal damage biomarkers such as IFABP, LFABP, 
and TFF3 are potential and powerful diagnostic tools to determine the intestinal damage caused by an intestinal obstruction in calves with atresia coli. High IAP and lactate levels may serve as a signal for the development of intestinal injury. For the practical usefulness of these biomarkers, further studies on their prognostic importance and an evaluation of their economic case are needed.

Conflict of Interests Statement: The authors declare that there is no conflict of interests regarding the publication of this article.

Financial Disclosure Statement: This study was supported by a grant received from the Selcuk University, Scientific Research Project Office (Project number: 15401147).

Animal Rights Statement: The research procedure was carried out with the approval of the Institutional Ethics Committee of the Faculty of Veterinary Medicine, Selcuk University (No. 2015/02).

\section{References}

1. Alkan F., Koc Y., Ceylan C.: The surgical repair of calves with atresia coli. Indian Vet J 2002, 79, 841-843.

2. Brown C.C., Baker D.C., Barker I.K.: Alimentary system. In: Palmer's Pathology of Domestic Animals, edited by G.M. Maxie, Elsevier-Saunders, Edinburgh, 2007, pp. 85-86.

3. Chang R.M., Wen L.Q., Chang J.X., Fu Y.R., Jiang Z.P., Chen S.: Repair of damaged intestinal mucosa in a mouse model of sepsis. World J Emerg Med 2013, 4, 223-228.

4. Constable P.D.: Acute intestinal obstructions in large animals. In: Merck Veterinary Medicine Manual, edited by C.M. Kahn, Merck \& Co, Whitehouse Station. 2010, pp. 224-225

5. Constable P.D., Rings D.M., Hull B.L.: Atresia coli in calves: etiopathogenesis and surgical management. Bovine Pract 1999, 33, 70-73.

6. Correa-Martín L., Párraga E., Sánchez-Margallo F.M., Latorre R., López-Albors O., Wise R., Castellanos G.: Mechanical intestinal obstruction in a porcine model: effects of intra-abdominal hypertension. A preliminary study. PloS One 2016, 11, e0148058.

7. Coskun A., Aydogdu U., Altan S., Erol M., Erol H., Guzelbektes H., Sen I.: Hematologic, blood gas, cardiac biomarkers, and serum biochemical parameters in calves with atresia coli and theirs relationship with prognosis. Acta Sci Vet 2017, 45, 1-6.

8. Cronk D.R., Houseworth T.P., Cuadrado D.G., Herbert G.S., McNutt P.M., Azarow K.S.: Intestinal fatty acid binding protein (I-FABP) for the detection of strangulated mechanical small bowel obstruction. Curr Surg 2006, 63, 322-325.

9. De Backer D.: Abdominal compartment syndrome. Crit Care 1999, 3, 103-104.

10. Derikx J.P.M., Evennett N.J., Degraeuwe P.L., Mulder T.L., van Bijnen A.A., van Heurn L.W., Buurman W.A., Heineman E.: Urine based detection of intestinal mucosal cell damage in neonates with suspected necrotizing enterocolitis. Gut 2007, 56, 1473-1475.

11. Furuta G.T., Turner J.R., Taylor C.T., Hershberg R.M., Comerford K., Narravula S., Colgan S.P.: Hypoxia-inducible factor 1-dependent induction of intestinal trefoil factor protects barrier function during hypoxia. J Exp Med 2001, 193, 1027-1034.
12. Goldberg R.F., Austen W.G. Jr, Zhang X., Munene G., Mostafa G., Biswas S., McCormack M., Eberlin K.R., Nguyen J.T., Tatlidede H.S., Warren H.S., Narisawa S., Millán J.L., Hodin R.A.: Intestinal alkaline phosphatase is a gut mucosal defense factor maintained by enteral nutrition. Proc Natl Acad Sci 2008, 105, 3551-3556.

13. Holmes J.H., Lieberman J.M., Probert C.B., Marks W.H., Hill M.E., Paull D.L., Hall R.A.: Elevated intestinal fatty acid binding protein and gastrointestinal complications following cardiopulmonary bypass: a preliminary analysis. J Surg Res 2001, 100, 192-196.

14. Kanda T., Fujii H., Tani T., Murakami H., Suda T., Sakai Y., Ono T., Hatakeyama K.: Intestinal fatty acid-binding protein is a useful diagnostic marker for mesenteric infarction in humans. Gastroenterology 1996, 110, 339-343.

15. Kanda T., Tsukahara A., Ueki K., Sakai Y., Tani T., Nishimura A., Yamazaki T., Tamiya Y., Tada T., Hirota M., Hasegawa J., Funaoka H., Fujii H., Hatakeyama K.: Diagnosis of ischemic small bowel disease by measurement of serum intestinal fatty acid-binding protein in patients with acute abdomen: a multicenter, observer-blinded validation study. J Gastroenterol 2011, 46, 492-500.

16. Katoh M.: Trefoil factors and human gastric cancer. Int J Mol Med 2003, 12, 3-9.

17. Kittaka H., Akimoto H., Takeshita H., Funaoka H., Hazui H., Okamoto M., Ohishi Y.: Usefulness of intestinal fatty acidbinding protein in predicting strangulated small bowel obstruction. PloS One 2014, 9, e99915.

18. Koç Y., Alkan F., Ceylan C., Birdane F.: Evaluation of clinical and surgical approaches in 22 calves with atresia coli. Vet Bil Derg 2001, 17, 27-34.

19. Lallès J.P.: Intestinal alkaline phosphatase: multiple biological roles in maintenance of intestinal homeostasis and modulation by diet. Nutr Rev 2010, 68, 323-332.

20. Lapsekili E., Menteş Ö., Balkan M., Günal A., Yaman H., Kozak O., Peker Y.: Role of alkaline phosphatase intestineisomerase in acute mesenteric ischemia diagnosis. TJTES, 2016, $22,115-120$

21. Ng E.W., Poon T.C., Lam H.S., Cheung H.M., Ma T.P., Chan K.Y., Wong R.P., Leung K.T., Lam M.M., Li K., Ng P.C.: Gut-associated biomarkers LFABP, IFABP, and TFF3 and LIT score for diagnosis of surgical necrotizing enterocolitis in preterm infants. Ann Surg 2013, 258, 1111-1118.

22. Pelsers M.M., Hermens W.T., Glatz J.F.: Fatty acid-binding proteins as plasma markers of tissue injury. Clin Chim Acta 2005, 352, 15-35.

23. Relja B., Szermutzky M., Henrich D., Maier M., de Haan J.J., Lubbers T., Buurman W.A., Marzi I.: Intestinal-FABP and liverFABP: Novel markers for severe abdominal injury. Acad Emerg Med 2010, 17, 729-735.

24. Riggle K.M., Rentea R.M., Welak S.R., Pritchard K.A. Jr., Oldham K.T., Gourlay D.M.: Intestinal alkaline phosphatase prevents the systemic inflammatory response associated with necrotizing enterocolitis. J Surg Res 2013, 180, 21-26.

25. Sakamoto K., Kanda T., Bamba T., Funaoka H., Kosugi S., Yajima K., Ishikawa T.: Serum intestinal fatty acid binding protein in patients with small bowel obstruction citations. Sci Res 2013, 4, 302-307.

26. Schierz I.A.M., Giuffrè M., Piro E., Ortolano R., Siracusa F., Pinello G., Corsello G.: Predictive factors of abdominal compartment syndrome in neonatal age. Am J Perinatol 2014, 31, 49-54.

27. Srivastava S., Kedia S., Kumar S., Pratap Mouli V., Dhingra R., Sachdev V., Tiwari V., Kurrey L., Pradhan R., Ahuja V.: Serum human trefoil factor 3 is a biomarker for mucosal healing in ulcerative colitis patients with minimal disease activity. J Crohns Colitis 2015, 9, 575-579.

28. Syed M., Shanks R.D.: Atresia coli inherited in Holstein cattle. J Dairy Sci 1992, 75, 1105-1111.

29. Uzun O., Turkmen S., Eryigit U., Mentes E.A., Turkyilmaz S., Turedi S., Karahan S.C., Gunduz A.: Can intestinal fatty acid 
binding protein (IFABP) be a marker in the diagnosis of abdominal pathology? Turk J Emerg Med 2014, 14, 99-103.

30. Vieten D., Corfield A., Carroll D., Ramani P., Spicer R.: Impaired mucosal regeneration in neonatal necrotising enterocolitis. Pediatr Surg Int 2005, 21, 153-160.
31. Zeyneloglu P.: Abdominal compartment syndrome. Turk J Intense Care 2015, 13, 5-15.

32. Zurek J., Kýr M., Vavřina M., Fedora M.: Trefoil factor 3 as a marker of intestinal cell damage during sepsis. Open Med $2015,10,261-266$. 